\title{
Trypping up tumors
}

\author{
By Tracey Baas, Senior Editor
}

A major limitation of many cancer immunotherapies is their inability to trigger a potent $\mathrm{CD} 8^{+} \mathrm{T}$ cell response, which is critical for battling different types of tumors. Brazilian and U.S. researchers may have solved the problem by using an attenuated form of the parasite Trypanosoma cruzi to express and deliver cancer antigens. ${ }^{1}$ Their prototype immunotherapy stimulated a potent antitumor response in mice, and the team now is taking steps to ensure the safety of the platform.

T. cruzi has a number of features that make it stand out as a potential cancer immunotherapy., ${ }^{2,3}$ First, T. cruzi is able to persist in host

"The benefits of this strain include the potential to get better cross-presentation of multiple epitopes across various human leukocyte antigen types. In addition, the parasites contain their own adjuvant, which eliminates the need for additional compounds to activate the immune system."

- Tibor Keler,

Celldex Therapeutics Inc. tissue and thus can support a sustained immune response. Second, the parasite presents antigen in a way that induces a strong $\mathrm{CD}^{+} \mathrm{T}$ cell response. Third, the parasite naturally expresses activating ligands of toll-like receptors (TLRs), which could make the use of adjuvants unnecessary.

The team set out to develop a prototype cancer immunotherapy that took advantage of these characteristics. Because $T$. cruzi causes Chagas disease, the researchers' first priority was choosing a noninfectious strain that was sufficiently attenuated to preclude development of the disease when using it to deliver antigen. ${ }^{4}$

Next, the team had to identify a good tumor antigen. They settled on human cancer/testis antigen 1B (CTAG1B; NY-ESO-1), which is known to induce cellular and humoral immunity and is expressed at a frequency of $20 \%-40 \%$ in melanoma, lung, breast, ovarian and bladder cancers.

The cancer immunotherapy prototype consisted of the attenuated T. cruzi strain engineered to express about 40 copies of the NY-ESO-1 gene.

In mice, a homologous prime and boost protocol with the immunotherapy led to complete protection against a subsequent challenge with melanoma cells, whereas all animals receiving only the NY-ESO-1 antigen plus a TLR agonist eventually developed melanoma and died following the challenge.

The researchers also used the compound as a therapeutic, which is how it would most likely be used in the clinical setting. In mice implanted with melanoma, fibrosarcoma or colon adenoma cells, three doses of the immunotherapy delayed tumor growth and prolonged survival compared with the attenuated strain not expressing NY-ESO-1. There were no cases of parasitemia or T. cruzi infection at 90 days postimmunization.

In peripheral blood mononuclear cells from healthy volunteers, the immunotherapy induced potent NY-ESO-1-specific CD4 ${ }^{+}$and $\mathrm{CD} 8^{+} \mathrm{T}$ cell responses.

Principal investigator Ricardo Gazzinelli told SciBX it should be possible to replace some of the 40 copies of NY-ESO- 1 with genes encoding additional cancer antigens, thus generating a polyvalent cancer immunotherapy.

"We have not identified what would be the ideal combination of tumor antigens to make a polyvalent vaccine but are working towards that," said Gazzinelli, who is professor of infectious disease and immunology at both the Federal University of Minas Gerais and the University of Massachusetts Medical School.

"The biggest advantage of NY-ESO-1-expressing T. cruzi is the fact that all the elements that are necessary to induce a good, long-lasting immune response are present in the nonvirulent parasite," said Santuza Teixeira, one of the authors of the paper and an associate professor at the Federal University of Minas Gerais. "The large majority of cancer vaccine candidates that have been tested so far are composed only of the antigen plus one or two adjuvants that can boost the immune system."

Results were published in the Proceedings of the National Academy of Sciences. The team included researchers from the Ludwig Institute for Cancer Research's New York branch, located at the Memorial SloanKettering Cancer Center.

Tibor Keler, founder, SVP and CSO of Celldex Therapeutics Inc., noted that "the benefits of this strain include the potential to get better cross-presentation of multiple epitopes across various human leukocyte antigen types," he said. "In addition, the parasites contain their own adjuvant, which eliminates the need for additional compounds to activate the immune system."

The ability of HLAs to cross-present tumor antigens to cytotoxic $\mathrm{T}$ cells is key to the success of cancer immunotherapies. Crosspresentation allows immunotherapies to stimulate and expand the specific population of T cells capable of identifying and directly killing the tumor cells. It also leads to a lasting response in which $\mathrm{T}$ cells continue to patrol for tumor cells expressing the relevant antigen.

Celldex's CDX-1401, a fusion protein consisting of a human mAb with specificity for a dendritic cell receptor linked to NY-ESO-1, is in Phase I/II testing to treat solid tumors.

\section{Parasites with benefits}

Researchers were concerned about the potential of the attenuated T. cruzi strain to revert to a more virulent form.

"Much work is needed to make this strategy ready for the clinic," said Teixeira. "Although the noninfectious clone has been passaged in the laboratory for about a decade and remains stable, there are always concerns about reversion to an infectious state. My lab is most interested in gaining a better understanding of the basis of the nonvirulence of the T. cruzi strain used as the candidate vaccine. We are [using] comparative 


\section{ANALYSIS}

genomics to try to identify the genomic differences between the virulent and this nonvirulent strain."

"Safety and immunogenic issues will need to be evaluated in nonhuman primates," added Mauricio Martins Rodrigues, a professor of microbiology, immunology and parasitology at the Federal University of São Paulo.

Gazzinelli acknowledged that the immunotherapy will need to be tested in primates but said his group's near-term focus is immunogenicity tests of the melanoma prototype vaccine in dogs, as well as scaling up GMP production.

For additional safety, his group is working on a next-generation vaccine that can prevent possible reverted T. cruzi strains from causing infection through the use of a suicide gene that would kill the parasite in response to the administration of a small molecule.

"We generated a transgenic parasite that expresses not only the cancer antigen NY-ESO-1 but also a suicide gene encoding herpes simplex virus thymidine kinase (HSV-tk). The transgenic parasite can now be cleared by administering acyclovir, the traditional antiviral therapy for HSV. Acyclovir is a prodrug that when processed by thymidine kinase becomes toxic and kills the parasite. We have done the experiments and it worked well in vitro. We are trying now to demonstrate this in mice," he said.
The Federal University of Minas Gerais and the Ludwig Institute for Cancer Research have filed for a patent covering a prophylactic and therapeutic NY-ESO-1-expressing T. cruzi-based vaccine in Brazil and the U.S. The work is available for licensing.

Baas, T. SciBX 5(2); doi:10.1038/scibx.2012.33

Published online Jan. 12, 2012

\section{REFERENCES}

1. Junqueira, C. et al. Proc. Natl. Acad. Sci. USA; published online Nov. 23, 2011; doi:10.1073/pnas.1110030108

Contact: Ricardo T. Gazzinelli, Federal University of Minas Gerais, Minas Gerais, Brazil e-mail: ritoga@cpqrr.fiocruz.br

2. Gazzinelli, R.T. \& Denkers, E.Y. Nat. Rev. Immunol. 6, 895-906 (2006)

3. Junqueira, C. et al. Expert Rev. Mol. Med. 12, e29 (2010)

4. Lima, M.T. et al. Parasitol. Res. 77, 77-81 (1991)

\section{COMPANIES AND INSTITUTIONS MENTIONED}

Celldex Therapeutics Inc. (NASDAQ:CLDX), Needham, Mass. Federal University of Minas Gerais, Minas Gerais, Brazil Federal University of São Paulo, São Paulo, Brazil Ludwig Institute for Cancer Research, New York, N.Y. Memorial Sloan-Kettering Cancer Center, New York, N.Y. University of Massachusetts Medical School, Worcester, Mass. 